\title{
A quantum particle in a box with moving walls
}

\author{
Sara Di Martino ${ }^{1}$, Fabio Anzà ${ }^{2}$, Paolo Facchi ${ }^{3,4}$, Andrzej \\ Kossakowski $^{5}$, Giuseppe Marmo ${ }^{6,7}$, Antonino Messina ${ }^{2}$, \\ Benedetto Militello ${ }^{2}$ and Saverio Pascazio ${ }^{3,4}$ \\ ${ }^{1}$ Dipartimento di Matematica, Università di Bari, I-70125 Bari, Italy \\ ${ }^{2}$ Dipartimento di Fisica e Chimica, Università di Palermo, I-90123 Palermo, Italy \\ ${ }^{3}$ Dipartimento di Fisica and MECENAS, Università di Bari, I-70126 Bari, Italy \\ ${ }^{4}$ INFN, Sezione di Bari, I-70126 Bari, Italy \\ ${ }^{5}$ Institute of Physics, Nicolaus Copernicus University, 87-100 Toruń, Poland \\ ${ }^{6}$ Dipartimento di Fisica and MECENAS, Università di Napoli "Federico II", I-80126 \\ Napoli, Italy \\ ${ }^{7}$ INFN, Sezione di Napoli, I-80126 Napoli, Italy
}

\begin{abstract}
We analyze the non-relativistic problem of a quantum particle that bounces back and forth between two moving walls. We recast this problem into the equivalent one of a quantum particle in a fixed box whose dynamics is governed by an appropriate time-dependent Schrödinger operator.

PACS numbers: 03.65.-w; 03.65.Db; 03.65.Xp
\end{abstract}

Keywords: Quantum boundary conditions, Time dependent Schrödinger operators 


\section{Introduction}

In 1949 Enrico Fermi [1] proposed a model for cosmic ray production in which a particle moves in inhomogeneous magnetic fields. This problem was reconsidered by Ulam [2], who introduced the so-called Fermi accelerator in which a ball bounces back and forth between two oscillating walls. He numerically discovered that such a system displays a very irregular behavior, and since then it has become a paradigmatic model to illustrate the chaotic nature of stochastic trajectories in systems with two degrees of freedom [3]. A non-relativistic quantum version of this problem was first studied, to the best of our knowledge, by Doescher and Rice [4], who analyzed the case in which one of the walls is static while the second one moves with a linear velocity. A number of articles followed, studying the problem from different aspects. In particular, the solvability of the model was investigated in a series of papers by Dembiński, Makowski, et al $[5,6,7,8]$ who exhibited several exact and perturbative solutions. Moreover, Dodonov et al [9] analyzed uniformly moving walls, including the cases of adiabatic motion and almost sudden changes of the wall position, while Munier et al [10] and Konishi and Paffuti [11] investigated the behaviour of the average energy. The problem in higher dimensions was considered by Lenz et al [12] and by Del Campo and Boshier [13], who focused on BoseEinstein condensates and experimental realizations.

Generally speaking, problems of this sort are tackled by searching solutions of the time-dependent Schrödinger equation related to the appropriate (time-dependent or static) Hamiltonian satisfying time-dependent boundary conditions. Such a direct approach, however, overviews the structural difficulty that the problem is ill-posed in its own, since one is requested to find solutions belonging to a time-dependent Hilbert space. In other words, the constraints characterizing the physical problem under scrutiny (no accessibility into time-dependent regions of the line) are mathematically taken into account by writing down a dynamical equation defined within a continuously timechanging space domain. This is an ill-posed problem since $t$ is among the independent variables of the same equation. In this direction, a rigorous analysis of time-dependent Schrödinger operators and/or time-dependent domains can be found in the work of Yajima [14, 15], Dell'Antonio et al [16] and Posilicano et al [17, 18].

The quantum bouncer problem is also interesting in the general context of quantum boundary conditions. Indeed, the behavior of the wave function at the boundary must reflect the quantum mechanical necessity to define observables in terms of self-adjoint operators [19, 20, 21, 22]. The most commonly used boundary conditions in physics are Dirichlet (vanishing wave function at the boundary), Neumann (vanishing normal derivative), and periodic ones. The interplay among different boundary conditions, with focus on an interval of the real line, was analyzed in [23, 24] and their role and importance has been recently stressed in an interesting article [25], where varying boundary conditions are viewed as a model of spacetime topology change. Notable applications arise in the context of the Casimir effect and its dynamical version, giving rise to photon generation in a microwave cavity with time-dependent boundary 
conditions $[26,27]$.

In this paper, we recast the problem of a quantum particle in a box with moving walls into the problem of a quantum particle placed in a box with fixed walls and governed by a time-dependent Schrödinger operator. Important properties of the system will be analyzed, such as the rate of energy change. The backbone of the article is the following. In section 2 we introduce the physical problem and the relevant Hamiltonian model, and introduce the unitary transformation that enables us to recast the original problem into a problem with fixed boundary conditions. In section 3 we derive the rate equation for the energy of the particle, singling out the role of contact terms with the walls, expressed by the presence of time derivatives of the wave function at the positions of the two walls. In section 4 we complete our treatment by including a potential acting on the particle. Finally, in section 5 we give some concluding remarks and discuss future perspectives.

\section{Hamiltonian Model}

\subsection{A particle bouncing between moving walls}

Consider a free massive particle in a one-dimensional box delimited by two walls at positions $a=-l / 2+d$ and $b=l / 2+d$. The box has therefore width $l>0$ and is centered at $d \in \mathbb{R}$, where $l$ and $d$ are functions of time, $l(t)$ and $d(t)$.

If the walls are still, the dynamics is described by the Schrödinger equation

$$
\mathrm{i} \hbar \frac{\mathrm{d}}{\mathrm{d} t} \psi(t)=\frac{p^{2}}{2 m} \psi(t)
$$

in the Hilbert space $L^{2}\left(I_{l, d}\right)$ of square integrable functions on the interval

$$
I_{l, d}=[a, b]=\left[d-\frac{l}{2}, d+\frac{l}{2}\right] \text {. }
$$

Here $p^{2}=-\hbar^{2} \frac{\mathrm{d}^{2}}{\mathrm{~d} x^{2}}$, acting on functions belonging to $\mathcal{H}^{2}\left(I_{l, d}\right)$, the Sobolev space of square integrable functions with square integrable second derivative, and vanishing at the ends (Dirichlet boundary conditions). Namely,

$$
D_{l, d}=\left\{\psi \in \mathcal{H}^{2}\left(I_{l, d}\right), \psi\left(d-\frac{l}{2}\right)=\psi\left(d+\frac{l}{2}\right)=0\right\} .
$$

However, when the walls are moving, the interpretation of an apparently innocuous equation as (1) requires some care. At first sight, if interpreted, as above, as an equation among different Hilbert spaces, it is meaningless. Indeed, the time derivative

$$
\frac{\mathrm{d}}{\mathrm{d} t} \psi(t)=\lim _{\epsilon \rightarrow 0} \frac{\psi(t+\epsilon)-\psi(t)}{\epsilon},
$$

would involve the sum of two vectors that belong in general to different Hilbert spaces $L^{2}\left(I_{l(t), d(t)}\right) \neq L^{2}\left(I_{l(t+\epsilon), d(t+\epsilon)}\right)$. Therefore, in order to correctly formulate the problem, one has to give a meaning to the time derivative. 
This can be accomplished by embedding $L^{2}\left(I_{l, d}\right)$ in the larger Hilbert space of a particle on a line $L^{2}(\mathbb{R})$ viewed as the direct sum

$$
L^{2}(\mathbb{R})=L^{2}\left(I_{l, d}\right) \oplus L^{2}\left(I_{l, d}^{c}\right),
$$

where $X^{c}=\mathbb{R} \backslash X$ denotes the complement of the set $X$. Thus, the kinetic energy operator in the common enlarged space is given by the Hamiltonian

$$
H_{0}(l, d)=\frac{p_{l, d}^{2}}{2 m}=-\frac{\hbar^{2}}{2 m} \frac{\mathrm{d}^{2}}{\mathrm{~d} x^{2}} \oplus_{l, d} 0,
$$

acting on wave functions of the form $\psi+\phi$ with $\psi \in D_{l, d}$ and $\phi \in L^{2}\left(I_{l, d}^{c}\right)$. A heuristic derivation of the Hamiltonian (6) from the Hamiltonian of a free particle on a line, with the moving walls implemented by a quantum Zeno dynamics [28], is given in the Appendix.

It is worth noticing that the direct sum decomposition both in (5) and in (6), in the case of moving walls, is in fact time-dependent. Therefore, one has to cope with a Schrödinger equation with a time-dependent Hamiltonian on a time-dependent domain. The common attack strategy of this kind of problems is to describe the evolution by unitarily equivalent Schrödinger operators acting on a common fixed domain. We will also be following this approach in the next sections.

\subsection{Reduction to constant boundary conditions (static domain)}

The intervals $I_{l, d}$ with $l>0$ can be mapped to a standard reference interval

$$
I=I_{1,0}=\left[-\frac{1}{2}, \frac{1}{2}\right]
$$

via a translation $x \rightarrow x-d$ followed by a dilation $x \rightarrow x / l$. These transformations of $\mathbb{R}$ can be lifted to transformations on $L^{2}(\mathbb{R})$, implemented by one-parameter (strongly continuous) unitary groups:

$$
(T(d) \psi)(x)=\psi(x-d), \quad(D(s) \psi)(x)=\mathrm{e}^{-s / 2} \psi\left(\mathrm{e}^{-s} x\right) .
$$

Let us therefore consider the unitary transformation

$$
U(l, d): L^{2}(\mathbb{R}) \rightarrow L^{2}(\mathbb{R}), \quad U(l, d)=D(\ln l)^{\dagger} T(d)^{\dagger}=D(-\ln l) T(-d),
$$

which acts as

$$
(U(l, d) \psi)(\xi)=\sqrt{l} \psi(l \xi+d) .
$$

This transformation obviously maps $L^{2}\left(I_{l, d}^{c}\right)$ onto $L^{2}\left(I^{c}\right)$, and, more interestingly, the domain (3) onto the fixed domain $D=U(l, d) D_{l, d}$

$$
D=\left\{\phi \in \mathcal{H}^{2}(I), \phi\left(-\frac{1}{2}\right)=\phi\left(\frac{1}{2}\right)=0\right\} \subset L^{2}(I),
$$

describing Dirichlet boundary conditions in a fixed box $I$. Moreover, the Hamiltonian (6) is mapped into

$$
H(l)=U H_{0} U^{\dagger}=\frac{1}{l^{2}} \frac{p^{2}}{2 m} \oplus 0=-\frac{1}{l^{2}} \frac{\hbar^{2}}{2 m} \frac{\mathrm{d}^{2}}{\mathrm{~d} x^{2}} \oplus 0,
$$


with fixed domain $D \oplus L^{2}\left(I^{c}\right)$.

It is worth noticing that the unitary transformation (9) yields constant boundary conditions only when the latter involve only the wave function or only its derivative. This is the case of Dirichlet and Neumann boundary conditions but not the Robin ones [23],

$$
\psi^{\prime}\left(d \pm \frac{l}{2}\right)=\alpha \psi\left(d \pm \frac{l}{2}\right)
$$

Let us now take a closer look at $H(l)$. When one goes from the interval $I_{l, d}$ to the fixed interval $I$, through the unitary transformation $U$, the eigenfunctions of $H(l)$ are a rescaled version of the ones of $H_{0}$, while the eigenvalues scale by a factor $l^{-2}$ (the prefactor of $p^{2}$ ), since the distance between the walls change by a factor $l$. This is formally expressed by the transformation law of the momentum operator on the line:

$$
U(l, d) p U(l, d)^{\dagger}=D(\ln l)^{\dagger} p D(\ln l)=\frac{p}{l},
$$

since the translation group $T(d)=\exp (-\mathrm{i} d p / \hbar)$ commutes with its generator $p$.

From now on, for the reader's convenience, we will denote the (relevant parts of the) wave functions by $\psi(x)$ with $x \in I_{l, d}$ and by $\phi(\xi)$ with $\xi \in I$, in the frame with moving and fixed walls, respectively.

The Schrödinger equation in the frame with fixed box will contain additional geometrical terms, induced by the time-dependent transformation $\phi(t)=U(l(t), d(t)) \psi(t)$. Taking into account the Schrödinger equation in the original frame

$$
\mathrm{i} \hbar \frac{\mathrm{d}}{\mathrm{d} t} \psi(t)=H_{0}(l(t), d(t)) \psi(t),
$$

we obtain

$$
\mathrm{i} \hbar \frac{\mathrm{d}}{\mathrm{d} t} \phi=\left(H(l)+\mathrm{i} \hbar \dot{U}(l, d) U^{\dagger}(l, d)\right) \phi
$$

in an appropriate domain.

Let us compute the geometric contribution i $\hbar \dot{U} U^{\dagger}$ step by step. First of all the action of $\dot{U}=\mathrm{d} U(l(t), d(t)) / \mathrm{d} t$ on a test function $\psi$ :

$$
\left(\frac{\mathrm{d} U}{\mathrm{~d} t} \psi\right)(\xi)=\frac{\mathrm{d}}{\mathrm{d} t}(\sqrt{l} \psi(l \xi+d))=\frac{i}{2 \sqrt{l}} \psi(l \xi+d)+\sqrt{l}(\dot{l} \xi+\dot{d}) \psi^{\prime}(l \xi+d) .
$$

Therefore, being

$$
\left(U^{\dagger}(l, d) \phi\right)(x)=(T(d) D(\ln l) \phi)(x)=\frac{1}{\sqrt{l}} \phi\left(\frac{x-d}{l}\right),
$$

we have

$$
\mathrm{i} \hbar \frac{\mathrm{d} U}{\mathrm{~d} t} U^{\dagger} \phi(\xi)=\mathrm{i} \frac{\hbar}{2} \frac{j}{l} \phi(\xi)+\mathrm{i} \hbar\left(\frac{\dot{l}}{l} \xi+\frac{\dot{d}}{l}\right) \phi^{\prime}(\xi)
$$

that is

$$
\mathrm{i} \hbar \frac{\mathrm{d} U}{\mathrm{~d} t} U^{\dagger}=-\frac{i}{l}\left(x p-\mathrm{i} \frac{\hbar}{2}\right)-\frac{\dot{d}}{l} p
$$


with $x$ and $p$ the standard position and momentum operators. Thus, the geometric generator of the unitary transformation reads

$$
K(l, d)=\mathrm{i} \hbar \frac{\mathrm{d} U}{\mathrm{~d} t} U^{\dagger}=-\frac{\dot{l}}{l} x \circ p-\frac{\dot{d}}{l} p,
$$

where $A \circ B=(A B+B A) / 2$ is the symmetrized (Jordan) product of the operators $A$ and $B$, and the canonical commutation relation $[x, p]=\mathrm{i} \hbar$ has been used.

By plugging expressions (12) and (21) into Eq. (16), we finally obtain the expression of the Schrödinger equation for the transformed state in the reference frame with fixed walls:

$$
\mathrm{i} \hbar \frac{\mathrm{d}}{\mathrm{d} t} \phi=(H(l)+K(l, d)) \phi=\left(\frac{1}{l^{2}} \frac{p^{2}}{2 m}-\frac{\dot{l}}{l} x \circ p-\frac{\dot{d}}{l} p\right) \phi .
$$

Apart from the term $p^{2} /\left(2 m l^{2}\right)$ that we discussed above, in this new Schrödinger equation we find two geometric terms. Both of them are due to the fact that application of the unitary transformation fixes the walls. This consists on a translation $T(d(t))=\exp (-\mathrm{i} d(t) p / \hbar)$, generated by the momentum operator $p$ with domain $\mathcal{H}^{1}(\mathbb{R})$, and a dilation $D(\ln l(t))=\exp (-\mathrm{i} \ln l(t) x \circ p / \hbar)$, generated by the virial operator $x \circ p$ on its maximal domain in $L^{2}(\mathbb{R})$. Incidentally, we notice that the emergence of the virial operator in Eq. (22) was recently advocated by Jarzynski [29] on the basis of symmetry considerations.

Let us finally show that the fixed domain $D$ is a domain of self-adjointness for the total Hamiltonian in $(22)$. Notice that $K(l, d)$ is relatively bounded with 0 relative bound with respect to $H(l)$, for every $d \in \mathbb{R}$ and $l>0$. Indeed, $p$ is $p^{2}$-bounded on $D$ and $\|x p \phi\| \leq\|p \phi\| / 2$ for any $\phi \in D$. Thus, since $K(l, d)$ with domain $D$ is a symmetric operator, by the Kato-Rellich theorem the total Hamiltonian $H(l)+K(l, d)$ with domain $D(H(l)+K(l, d))=D(H(l))=D$ is self-adjoint.

Since for any pair of differentiable functions $d(t)$ and $l(t)$, with $l(t)>l_{0}$, for some $l_{0}>0, t \mapsto H(l(t))+K(l(t), d(t))$ is a one-parameter family of Schrödinger operators on a common domain of self-adjointness $D$, by standard abstract methods (see e.g. Theorem X.70 in [20]) the time-dependent Schrödinger equation (22) is well defined for any initial condition $\phi(0) \in D$, and yields a unique unitary propagator.

Equation (22) is a central result of this paper. We now check its consequences on some interesting physical situations.

\subsection{Some Examples}

Consider the case in which there is only a translation of the walls without dilation:

$$
i=0, \quad d(t)=d_{0}+v t .
$$

The Schrödinger operator in the static reference frame, by assuming $l=1$, is

$$
H+K=\frac{p^{2}}{2 m}-v p
$$


which is exactly the Hamiltonian transformed according to the Galilean transformation, as expected.

On the other hand, if we consider the case (constant acceleration $g$ )

$$
i=0, \quad d(t)=d_{0}+\frac{1}{2} g t^{2},
$$

we find:

$$
H=\frac{p^{2}}{2 m}-g t p
$$

(taking again $l=1$ ). In such a case, by applying the gauge (unitary) transformation

$$
G(t): L^{2}\left(-\frac{1}{2}, \frac{1}{2}\right) \rightarrow L^{2}\left(-\frac{1}{2}, \frac{1}{2}\right): \phi \mapsto \chi=G(t) \phi
$$

with

$$
\chi(\xi)=(G(t) \phi)(\xi)=\mathrm{e}^{\frac{i}{\hbar}\left(m g \xi t-\frac{1}{6} m g^{2} t^{3}\right)} \phi(\xi),
$$

the domain $D$ is left invariant, $G(t)(D)=D$, and the Hamiltonian becomes

$$
G(t) H G^{\dagger}(t)=\frac{p^{2}}{2 m}-\frac{1}{2} m g^{2} t^{2} .
$$

Moreover,

$$
\mathrm{i} \hbar \dot{G}(t) G^{\dagger}(t)=-m g x+\frac{1}{2} m g^{2} t^{2}
$$

so that the new Schrödinger equation is

$$
\mathrm{i} \hbar \frac{\mathrm{d}}{\mathrm{d} t} \phi(t)=\left(\frac{p^{2}}{2 m}-m g x\right) \phi(t),
$$

and describes a particle in a constant gravitational field, in agreement with the equivalence principle. Notice, however, that the particle is in a box (a falling elevator).

\section{Energy rate equation}

Let us consider an initial state $\phi(0)$ at time $t=0$. It will evolve into the state $\phi(t)$ solution of the Schrödinger equation (22).

The energy of the system has a rate given by

$$
\dot{E}(t)=\frac{\mathrm{d}}{\mathrm{d} t}\langle\phi \mid H(l) \phi\rangle=\frac{\mathrm{i}}{\hbar}(\langle\phi \mid K H \phi\rangle-\langle K H \phi \mid \phi\rangle)+\langle\phi \mid \dot{H} \phi\rangle,
$$

with $K(l, d)$ given by $(21)$, with domain

$$
D(K)=\left\{f \in \mathcal{H}^{1}(I), \xi f^{\prime}(\xi) \in L^{2}(I)\right\}
$$

and $\phi \in D(K H)$, where

$$
D(K H)=\left\{f \in \mathcal{H}^{3}(I), f\left(-\frac{1}{2}\right)=f\left(\frac{1}{2}\right)=0\right\} .
$$

A double integration by parts gives

$$
\frac{\mathrm{d}}{\mathrm{d} t}\langle\phi(t) \mid H(l(t)) \phi(t)\rangle=-\frac{\hbar^{2}}{2 m l^{3}}\left[(\dot{l}(t) \xi+\dot{d}(t))\left|\phi^{\prime}(\xi, t)\right|^{2}\right]_{-\frac{1}{2}}^{\frac{1}{2}},
$$


with $\phi^{\prime}(\xi, t)=\partial_{\xi} \phi(\xi, t)$.

In the original frame, since $\phi^{\prime}(\xi, t)=l^{3 / 2} \psi^{\prime}(l \xi+d, t)$, this yields

$$
\dot{E}(t)=\frac{\mathrm{d}}{\mathrm{d} t}\left\langle\psi(t) \mid H_{0}(l(t), d(t)) \psi(t)\right\rangle=-\frac{\hbar^{2}}{2 m}\left[\dot{b}(t)\left|\psi^{\prime}(b(t), t)\right|^{2}-\dot{a}(t)\left|\psi^{\prime}(a(t), t)\right|^{2}\right],
$$

with $a=-l / 2+d$ and $b=+l / 2+d$ the positions of the walls. As expected, due to the movement of the walls, the energy change is due only to boundary terms depending on the velocities of the walls. Indeed, within the walls the problem is that of a free particle, so that the energy changes only because of the presence of the moving walls. The case

$d=\frac{l}{2}$ is the standard Fermi-Ulam accelerator model, where one of the two walls is still at $a=0$, while the other is at $b=l$. In such a case the rate (36) particularizes to the result heuristically found by Konishi and Paffuti [11].

\subsection{Two Examples}

Let us now consider two examples that are valid within the limits of validity of perfectly reflecting walls. Assume

$$
l=l_{0}+v t, \quad d=0 .
$$

In this case the Schrödinger equation in the reference frame with fixed walls becomes

$$
\mathrm{i} \hbar \frac{\mathrm{d}}{\mathrm{d} t} \phi=\frac{1}{l_{0}+v t}\left(\frac{1}{l_{0}+v t} \frac{p^{2}}{2 m}-v \xi \circ p\right) \phi,
$$

while the energy rate is

$$
\dot{E}(t)=-\frac{v}{\left(l_{0}+v t\right)^{3}} \frac{\hbar^{2}}{2 m} \frac{1}{2}\left[\left|\phi^{\prime}\left(\frac{1}{2}, t\right)\right|^{2}+\left|\phi^{\prime}\left(-\frac{1}{2}, t\right)\right|^{2}\right] .
$$

As another example, we take

$$
l=l_{0}+\sin (\omega t), \quad d=0 .
$$

The Schrödinger equation in the reference frame is

$$
\mathrm{i} \hbar \frac{\mathrm{d}}{\mathrm{d} t} \phi=\frac{1}{l_{0}+\sin (\omega t)}\left(\frac{1}{l_{0}+\sin (\omega t)} \frac{p^{2}}{2 m}+\omega \cos (\omega t) \xi \circ p\right) \phi,
$$

and the energy rate reads

$$
\dot{E}(t)=-\frac{\omega \cos (\omega t)}{\left(l_{0}+\sin (\omega t)\right)^{3}} \frac{\hbar^{2}}{2 m} \frac{1}{2}\left[\left|\phi^{\prime}\left(\frac{1}{2}, t\right)\right|^{2}+\left|\phi^{\prime}\left(-\frac{1}{2}, t\right)\right|^{2}\right] .
$$

\subsection{A Remark on domains}

In the derivation of the energy rate given above we have been somewhat cavalier about domains. Formally, the expression (32) is nothing but the expectation value of the commutator $[K, H]$ (plus the expectation of $\dot{H}$ ). However, as written there, that expression is valid in a larger domain than $D([K, H])$, contained in its form domain. We now show that such an extension is needed, since for functions in $D([K, H])$ the energy rate identically vanishes. 
Let us take a closer look at the domains by focussing on their relevant part in $L^{2}(I)$. The Hamiltonian $H$ is defined on the Dirichlet domain (11), $D(H)=D$, while (the restriction of) $K$ is defined on $D(K)=\mathcal{H}^{1}(I)$, so that the domain of the commutator is

$$
\begin{aligned}
D([K, H]) & =\{\phi \in D(K), K \phi \in D(H)\} \cap\{\phi \in D(H), H \phi \in D(K)\} \\
& =\left\{\phi \in \mathcal{H}^{3}(I), \phi\left(-\frac{1}{2}\right)=\phi\left(\frac{1}{2}\right)=0, \phi^{\prime}\left(-\frac{1}{2}\right)=\phi^{\prime}\left(\frac{1}{2}\right)=0\right\} .
\end{aligned}
$$

As a consequence, for every $\phi \in D([K, H])$, one gets from (35)

$$
\frac{\mathrm{d}}{\mathrm{d} t}\langle\phi(t) \mid H(l) \phi(t)\rangle=0
$$

This means that the space $D([K, H])$ is too small for our purposes so we have to consider a larger space, such as $D(K H)$ in (34), which is contained in the form domain of the commutator.

\section{Adding a potential}

In order to complete our treatment, we consider now the case in which the particle is confined in a moving and dilating box, subject to the action of a time-dependent potential. In such a situation, the Hamiltonian of the system is given by

$$
H_{1}(l, d, t)=\left(-\frac{\hbar^{2}}{2 m} \frac{\mathrm{d}^{2}}{\mathrm{~d} x^{2}}+V(x, t)\right) \oplus_{l, d} 0,
$$

on the domain $D_{l, d} \oplus L^{2}\left(I_{l, d}^{c}\right)$. We assume that $V(\cdot, t): I_{l, d} \rightarrow \mathbb{R}$ is a measurable function such that $H_{1}(l, d, t)$ is self-adjoint. For this purpose it suffices, e.g., that $V(\cdot, t) \in L^{2}\left(I_{l, d}\right)$ for every $t, l$, and $d$.

Under the action of the unitary transformation $U$, the potential is transformed in a simple way:

$$
\tilde{V}(\cdot, t)=U(l, d) V(\cdot, t) U^{\dagger}(l, d), \quad \tilde{V}(\xi, t)=V(l \xi+d, t) .
$$

The rate equation for the energy of the particle is given by

$$
\begin{aligned}
\dot{E}_{1}(t)=\frac{\mathrm{d}}{\mathrm{d} t}\left\langle\psi(t) \mid H_{1}(l, d, t) \psi(t)\right\rangle & =\frac{\mathrm{i}}{\hbar}(\langle\phi \mid K H \phi\rangle-\langle K H \phi \mid \phi\rangle)+\langle\phi \mid \dot{H} \phi\rangle \\
& +\frac{\mathrm{i}}{\hbar}(\langle\phi \mid K \tilde{V} \phi\rangle-\langle K \tilde{V} \phi \mid \phi\rangle)+\langle\phi \mid \dot{\tilde{V}} \phi\rangle .
\end{aligned}
$$

Since $\dot{\tilde{V}}=-\mathrm{i} / \hbar[K, \tilde{V}]+U \partial V / \partial t U^{\dagger}$, and considering Eq. (32), one gets

$$
\dot{E}_{1}(t)=\dot{E}(t)+\left\langle\phi\left|U \frac{\partial V}{\partial t} U^{\dagger}\right| \phi\right\rangle .
$$

It is worth noting that while a detailed evaluation of the mean value of $\mathrm{i} / \hbar[K, H]+\dot{H}$ gives rise to contact terms, in the case of $\mathrm{i} / \hbar[K, \tilde{V}]+\dot{\tilde{V}}$ no similar contributions appear. This is due to the fact that $H$ is a differential operator, while $\tilde{V}$ is simply a multiplication operator.

As a special case, let us consider the situation where $V$ is essentially a static potential, meaning that there exists a certain $\varphi \in L_{\mathrm{loc}}^{2}(\mathbb{R})$, independent of time, such 
that $V(x, t)=\varphi(x)$ for $x \in I_{l, d}$ and $V(x, t)=0$ elsewhere. In this case it turns out that the presence of the potential does not add extra terms to the energy variation rate. Formally, time derivatives of $V$ can produce $\delta$-functions at the border, but since the wave function satisfies Dirichlet boundary conditions, the net result of the integration in Eq. (48) is zero.

\section{Conclusions}

We have analyzed the problem of the non-relativistic quantum bouncer. Our focus has been on the very structure of the problem and on the possible difficulties of framing it in a rigorous mathematical framework. The first question that we have tackled is the correct formulation of the problem in mathematical terms, avoiding the use of equations defined in different Hilbert spaces at different times. To this end, we have used an extended Hilbert space and defined all the operators as zero outside the box and nonzero (with their standard action) inside the box. Subsequently, to effectively solve the relevant dynamical problem, we have exploited a unitary transformation that converts the original problem into the problem of a particle moving in a fixed box but governed by a time-dependent Hamiltonian. This has required a proper redefinition of the problem, from that of a system evolving under time-dependent boundary conditions into that of a system suitably evolving under fixed boundary conditions. In such a way, we have ended up with a family of time-dependent Hamiltonian operators with a common domain of self-adjointness. As a consequence, the time-dependent Schrödinger equation is welldefined for any initial vector in the common domain, and the existence of a unique unitary propagator is assured.

An interesting aspect of our analysis is the possibility to write down a general rate equation describing the energy change undergone by the particle. In fact, also from a classical point of view, a particle interacting with moving walls is subject to some variation of kinetic energy, and this has a quantum mechanical counterpart, well expressed by the contact terms in Eq. (35). Moreover, we provided a further development by considering the effects of a potential. The relevant new terms in the rate equation of the energy do not include contact terms.

A possible extension of our results concerns the analysis of different boundary conditions and/or boxes in two- and three-dimensional spaces.

\section{Appendix A. Building up the walls by a product formula}

We derive here the Schrödinger equation for the static frame, starting from the Schrödinger equation of a free particle on a line and constructing the moving walls as the limit of infinitely time-dependent spatial projections, a limit known as quantum Zeno dynamics [28]. Consider the projections

$$
P(t)=\chi_{(-l(t) / 2+d(t), l(t) / 2+d(t))}(x),
$$


$\chi_{A}$ being the characteristic function of the set $A$. Then $P(t)=U^{\dagger} P U$, with $P=\chi_{\left(-\frac{1}{2}, \frac{1}{2}\right)}$ the projection on the moving box. If we discretize time: $t_{k}=k t / N$ where $k=0, \ldots, N$, the evolution in the moving box is approximated by the operator $(\hbar=1)$ :

$$
\begin{aligned}
W_{N}(t) & =P\left(t_{N}\right) \mathrm{e}^{-\frac{\mathrm{i} T t}{N}} \cdots \mathrm{e}^{-\frac{\mathrm{i} T t}{N}} P\left(t_{1}\right) \mathrm{e}^{-\frac{\mathrm{i} T t}{N}} P\left(t_{0}\right) \\
& =U^{\dagger}\left(t_{N}\right) \prod_{k=1}^{N}\left[P U\left(t_{k}\right) \mathrm{e}^{-\frac{\mathrm{i} T t}{N}} U^{\dagger}\left(t_{k-1}\right) P\right] U(0),
\end{aligned}
$$

where $T=\frac{p^{2}}{2 m}$ is the kinetic energy operator on the whole line $\mathbb{R}$ and we used the notation $U\left(t_{k}\right)=U\left(l\left(t_{k}\right), d\left(t_{k}\right)\right)$. Now we formally compute [30]

$$
\begin{aligned}
P U\left(t_{k}\right) \mathrm{e}^{-\frac{\mathrm{i} T t}{N}} U^{\dagger}\left(t_{k-1}\right) P= & \exp \left(-\frac{\mathrm{i} t}{N} P\left(T\left(t_{k-1}\right)+\mathrm{i} \dot{U}\left(t_{k-1}\right) U^{\dagger}\left(t_{k-1}\right)\right) P\right) P \\
& +O\left(\frac{t^{2}}{N^{2}}\right),
\end{aligned}
$$

So that, in the limit $N \rightarrow \infty$

$$
W(t)=\lim _{N \rightarrow \infty} W_{N}(t)=U^{\dagger}(t) \mathcal{T} \mathrm{e}^{-\mathrm{i} \int_{0}^{t} P\left(T(s)+\mathrm{i} \dot{U}(s) U^{\dagger}(s)\right) P d s} P U(0),
$$

$\mathcal{T}$ denoting time-ordering. The evolution in the original frame is therefore

$$
\psi(t)=W(t) \psi(0),
$$

while in the static reference frame

$$
\phi(t)=U(t) W(t) U^{\dagger}(0) \phi(0)=\mathcal{T} \mathrm{e}^{-\mathrm{i} \int_{0}^{t} P\left(T(s)+\mathrm{i} \dot{U}(s) U^{\dagger}(s)\right) P d s} P \phi(0) .
$$

In conclusion, the Schrödinger equation in the frame with fixed box reads

$$
\mathrm{i} \dot{\phi}(t)=\left(H(t)+\mathrm{i} \dot{U}(t) U^{\dagger}(t)\right) \phi(t),
$$

where $H$ is the operator defined in Eq. (12).

\section{References}

[1] Fermi E 1949 Phys. Rev. 751169

[2] Ulam S M 1961 Proc. Fourth Berkeley Symposium on Mathematical Statistics and Probability vol 3 (Berkeley, CA: University of California Press) p 315

[3] Lichtenberg A J and Lieberman M A 1992 Regular and Chaotic Dynamics (NewYork: Springer)

[4] Doescher S W and Rice M H 1969 Am. J. Phys. 371246

[5] Makowski A J and Dembiński S T 1991 Phys. Lett. A 154217

[6] Makowski A J 1992 J. Phys. A: Math. Gen. 253419

[7] Dembiński S T, Makowski A J and Pepłowski P 1993 Phys. Rev. Lett. 701093

[8] Makowski A J 1996 J. Phys. A: Math. Gen. 296003

[9] Dodonov V V, Klimov A B and Nikonov D E 1993 J. Math. Phys. 343391

[10] Munier A, Burgan J R, Feix M and Fijalkow E 1981 J. Math. Phys. 22329

[11] Konishi K and Paffuti G 2009 Quantum Mechanics: A New Introduction (Oxford: Oxford University Press)

[12] Lenz F, Liebchen B, Diakonos F K and Schmelcher P 2011 New Journal of Physics 13103019

[13] Del Campo A and Boshier M G 2012 Sci. Rep. 2648

[14] Yajima K 1987 Commun. Math. Phys. 110415 
[15] Yajima K 1996 Commun. Math. Phys. 181605

[16] Dell'Antonio G F, Figari R and Teta A 2000 The Schrödinger equation with moving point interactions in three dimensions in Stochastic Processes, Physics and Geometry: New Interplays, I (Leipzig, 1999) (CMS Conference Proceedings vol 28) (American Mathematical Society: Providence, RI) pp 99-113

[17] Posilicano A 2007 Proc. Amer. Math. Soc. 1351785

[18] Dias N C, Posilicano A and Prata J N 2011 Commun. Pure Appl. Anal. 101687

[19] von Neumann J 1995 Mathematical Foundation of Quantum Mechanics (Princeton, NJ: Princeton University Press)

[20] Reed M and Simon B 1975 Methods of Modern Mathematical Physics: Fourier Analysis, Self Adjointness vol 2 (London: Academic Press)

[21] Peres A 2002 Quantum Theory: Concepts and Methods (New York: Kluwer)

[22] Galindo A and Pascual P 1990 Quantum Mechanics (Berlin: Springer)

[23] Asorey M, Ibort A and Marmo G 2005 Int. J. Mod. Phys. A 201001

[24] Asorey M, Facchi P, Marmo G and Pascazio S 2013 J. Phys. A: Math. Theor. 46102001

[25] Shapere A D, Wilczek F and Xiong Z 2012 Models of topology change arXiv:1210.3545 [hep-th]

[26] Wilson C M, Duty T, Sandberg M, Persson F, Shumeiko V and Delsing P 2010 Phys. Rev. Lett 105233907

[27] Lähteenmäki P, Paraoanu G S, Hassel J and Hakonen P J 2013 Proc. Natl. Acad. Sci. USA 110 4234

[28] Facchi P and Pascazio S 2008 J. Phys. A: Math. Theor. 41493001

[29] Jarzynski C 2013 Generating shortcuts to adiabaticity in quantum and classical dynamics arXiv:1305.4967 [quant-ph]

[30] Burgarth D, Facchi P, Giovannetti V, Nakazato H, Pascazio S and Yuasa K 2013 Non-Abelian Phases from a Quantum Zeno Dynamics arXiv:1305.6433 [quant-ph] 